\title{
The first success of glass eel production in the world: basic biology on fish reproduction advances new applied technology in aquaculture
}

\author{
Hirohiko Kagawa $\cdot$ Hideki Tanaka $\cdot$ \\ Hiromi Ohta · Tatsuya Unuma • \\ Kazuharu Nomura
}

(C) Springer Science+Business Media B.V. 2006

\begin{abstract}
The eel has long been esteemed as an important food fish in the world, especially in Japan, and has been used as an experimental fish for many fields of fish physiology. However, the decreases in eel resources have been a serious concern in recent years. The catches of glass eels as seedlings for aquaculture have shown a long-term decrease in both Europe and East Asia. To increase eel resources, the development of techniques for artificial induction of maturation and spawning and rearing their larvae have been eagerly desired. Recent progress of reproductive physiology of fish, especially mechanisms of oocyte maturation and ovulation in female and of spermatozoa maturation in male, facilitate to establish techniques for hormonal induction of maturation and spawning in sexually immature eels. With persistent effort to development of rearing techniques of larvae, we have first succeeded to produce glass
\end{abstract}

H. Kagawa $(\bowtie)$

Department of Biological Production and Environmental Science, Faculty of Agriculture, University of Miyazaki, Miyazaki 889-2192, Japan

e-mail: kagawa@cc.miyazaki-u.ac.jp

H. Tanaka $\cdot$ T. Unuma $\cdot$ K. Nomura

National Research Institute of Aquaculture, Nansei,

Mie 516-0193, Japan

H. Ohta

Department of Fisheries, School of Agriculture, Kinki

University, Nara 631-8505, Japan eel. These applied techniques are may contribute to understand the basic reproductive physiology of the eel.

Keywords Japanese eel · Artificial maturation · Oogenesis $\cdot$ Spermatogenesis $\cdot$ Artificial insemination - Larval rearing · Leptocephalus · Glass eel

\section{Introduction}

The eel has long been esteemed not only in Japan but also in European countries as an important food fish. The aquacultural production of Japanese eel, Anguilla japonica, in Japan is about 30,000 tons a year in recent years. Japan also imports a total of 80,000 tons of eel from mainly China and Taiwan, and other countries. Seedlings for eel aquaculture are totally dependent on glass eels, natural juveniles of eel which captured in estuaries. However, in both East Asia and Europe the catches of glass eels differ greatly from year to year and especially in the past 25 years, have been decreasing, resulting in a sharp rise in its price. Therefore, to maintain the natural glass eel resources and to obtain reliable supplies of glass eels for aquaculture, development of an artificially induced breeding procedure for eels has been eagerly desired.

Techniques for artificial breeding of the Japanese eel have been studied intensively since the 1960s. 
Yamamoto and Yamauchi (1974) first succeeded in obtaining fertilized eggs and larvae of the Japanese eel by hormone treatment, and preleptocephalus larvae were reared for 2 weeks (Yamauchi et al. 1976). Thereafter, many researchers have succeeded in obtaining eel larvae, but preleptocephalus larvae could not survive beyond the depletion of their yolk and oil droplet stores. Failures of production of the glass eel may be caused by incomplete techniques for inducing sexual maturation of both male and female Japanese eels and incomplete rearing techniques of larvae. Basic science on reproductive biology of fish has remarkably progressed, especially in the fields of endocrine mechanisms of oogenesis and spermatogenesis. Therefore, it is expected that information from the basic science facilitate to develop techniques for induction of sexual maturation and rearing techniques of the Japanese eel larvae. Therefore, in this paper, our recent researches on glass eel production, mainly on induction of sexual maturation, are reviewed. Using newly developed techniques, our group has succeeded for the first time to obtain glass eels.

\section{Maturation and ovulation}

Cultivated female eels or silver eels that migrate down river are sexually immature and their gonadosomatic index slightly increase to around $1-2 \%$ in fall and their ovaries contain oocytes at the oil stage or oocytes at the primary yolk globule stage (Kagawa et al. 1998). However, cultivated and silver eels do not mature and ovulate under normal culture conditions. Yamamoto and Yamauchi (1974) succeeded in inducing oocyte growth and obtaining full-grown oocytes in almost of all fish by repeated injections of salmon pituitary extracts. Since then, repeated injections of piscine pituitary (mainly carp or salmon pituitary) extracts are now routinely used for induction of vitellogenesis in female eels. However, the percentage of ovulated females is very low, and even if ovulated eggs are obtained, these eggs show low fertility and hatchability.

After 8-13 injections of salmon pituitary extracts (20 mg/fish/week), females have ovaries containing the full-grown oocytes which complete vitellogenesis and attain the migratory nucleus stage (about $750-800 \mu \mathrm{m}$ in diameter in Japanese eel, Ohta et al.
1997; Kagawa et al. 1998). However, their oocytes do not undergo maturation and become over-ripe with oocyte cytoplasmic degeneration in response to further injections of salmon pituitary extracts.

After the oocytes complete vitellogenesis, oocyte maturation occurs prior to ovulation and is a prerequisite for successful fertilization; it consists of breakdown of germinal vesicle (GVBD), the resumption of meiosis, and oocyte cytoplamic maturation (see Nagahama et al. 1995). These processes of oocyte maturation are regulated by the pituitary gonadotropin (primary endocrine factor responsible for triggering meiotic maturation) and maturationinducing steroid which is synthesized in the ovarian follicle under the influence of gonadotropin. The maturation-inducing steroid directly acts on the oocyte to initiate the process of oocyte maturation (Nagahama et al. 1995). The maturation-inducing steroid ( 17, 20ß-dihydroxy-4-pregnen-3-one, DHP) of a salmonid fish, amago salmon (Oncorhynchus rhodurus), was isolated for the first time in vertebrate species (Nagahama and Adachi 1985) and thereafter DHP has been found to be the most effective steroid in inducing oocyte maturation of many fish species. Yamauchi and Yamamoto (1982) reported that in vitro administration of DHP into the incubation medium induced oocyte maturation in the Japanese eel. From these fundamental researches, it is expected that in vivo administration of DHP could induce oocyte maturation and ovulation.

For application of DHP on artificial induction of maturation and ovulation in the Japanese eel, other valuable information on oocyte maturation and ovulation have been obtained. From basic researches of red seabream (Kagawa et al. 1994a, b; Patino et al. 2001), we have found that process of oocyte maturation consists of two critical periods. During the first stage of maturation, oocytes acquire ability to respond to maturation inducing steroid, whereas in the second stage the follicles produce maturation-inducing steroid and, consequently, the oocyte undergo germinal vesicle breakdown. In vitro incubation of oocyte of the Japanese eel at various developmental stages revealed (Kagawa et al. 1995) that oocytes undergo GVBD in response to both $17 \alpha$-hydroxyprogesterone and DHP when oocytes diameter attain the migratory nucleus stage (approximately $700-800 \mu \mathrm{m}$ in diameter). These results suggest that oocytes acquire the ability to respond to maturation- 
inducing steroid (maturational competence) at the migratory nucleus stage. However, the production of maturation-inducing steroid is not induced by further injections of salmon pituitary extracts, possibly due to a lack of precursor synthesis (Ijiri et al. 1995), resulting in failure of induction of maturation and ovulation by injection of salmon pituitary extracts. Our data also indicate that DHP can induce not only in vitro maturation but also in vitro ovulation (Kagawa et al. 2003). Moreover, DHP-induced in vitro ovulation occurred earlier in larger oocytes more than $860 \mu \mathrm{m}$ in diameter compared to smaller oocytes less than $860 \mu \mathrm{m}$ in diameter (Fig. 1). Data obtained from one female eel show that ovulation rates rapidly decreased after oocytes attain more than $860 \mu \mathrm{m}$ in diameter (Fig. 2). These data suggest that not only oocyte maturation but also ovulation can be induced by in vivo administration of DHP, when female eels have ovaries containing maturational competent oocytes at the migratory nucleus stage.

From these in vitro experiments, the following techniques of induction of maturation and ovulation were developed (Fig. 3). Female eels that possess oocytes at the migratory nucleus stage (approximately $800-850 \mu \mathrm{m}$ in diameter) were injected with salmon pituitary extracts $(20 \mathrm{mg} / \mathrm{fish})$ as a priming dose followed $24 \mathrm{~h}$ later by an injection of DHP ( $2 \mu \mathrm{g} / \mathrm{g}$ body weight) (Ohta et al. 1996a; Kagawa 2003). Injection of DHP succeeded in inducing oocyte maturation and ovulation in almost all females

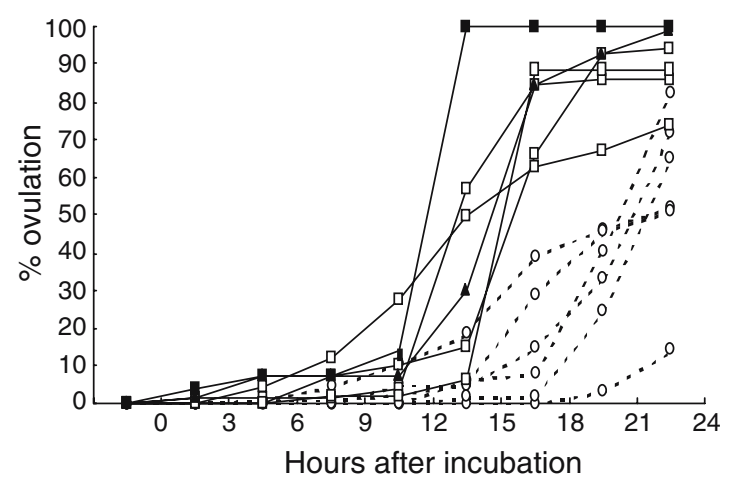

Fig. 1 Effects of 17, 20 $\beta$-dihydroxy-4-pregnen-3-one (DHP) on in vitro ovulation in the Japanese eel. Oocytes with follicular layers obtained from female eels were incubated in $1 \mathrm{ml} \mathrm{L}-15$ incubation media with DHP (100 ng/ml). Dotted line and solid line indicate changes in $\%$ ovulation of oocytes less than $860 \mu \mathrm{m}$ in diameter and more than $860 \mu \mathrm{m}$ in diameter respectively

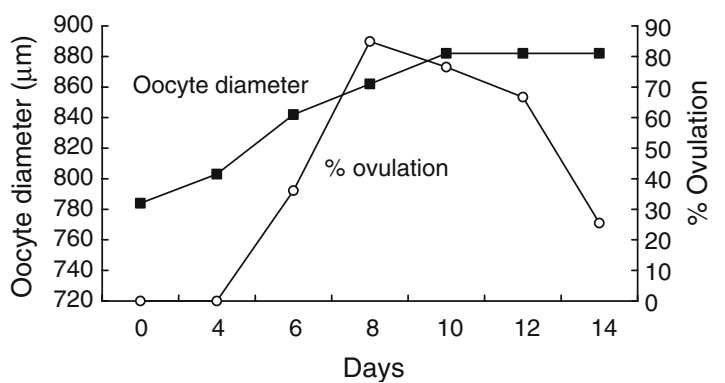

Fig. 2 Changes in oocyte diameter and in vitro ovulation rates of oocytes with follicle layers obtained from one female Japanese eel. Oocytes with follicle layers sequentially obtained from one female which was induce by repeated injection of salmon pituitary extracts were incubated in L-15 incubation media with DHP (100 ng/ml). Ovulation rates were observed $24 \mathrm{~h}$ after incubation

used in the experiments. Fertilization and hatching rates are approximately 60 and $40 \%$ respectively (Kagawa et al. 1997). Both fertilization and hatching rates of ovulated females decreased rapidly to almost $0 \%$ by $6 \mathrm{~h}$ after ovulation, suggesting that artificial fertilization must be carried out immediately after ovulation in order to obtain good quality eggs (Ohta et al. 1996a).

\section{Induction of spermatogenesis and spermiation}

Cultivated male Japanese eels are also sexually immature and do not mature under normal culture conditions. Injecting them with human chorionic gonadotropin (HCG) can easily induce maturation (Miura et al. 1991) and spermiation (Ohta et al. 1996b). After 6 weekly injections of HCG at a dose of $1 \mathrm{IU} / \mathrm{g} \mathrm{BW}$, most fish spermiate small volumes of milt, and milt volume increase and become stable after 11 th injection. However, there are major differences in the extent of maturity induced in individual fish after the same treatment with HCG. The effectiveness of HCG injection in inducing spermatogenesis is to be a considerable extent influenced by individual difference in the rates of HCG clearance among fish (Ohta and Tanaka 1997). Moreover, individual differences in the rate of sperm motility are significant problems among hormonally matured males, ranging between less than $10 \%$ and more than $90 \%$ (Ohta et al. 2001), leading to an obvious varying degree of successful 
Male

(B.W. 200-300 g)

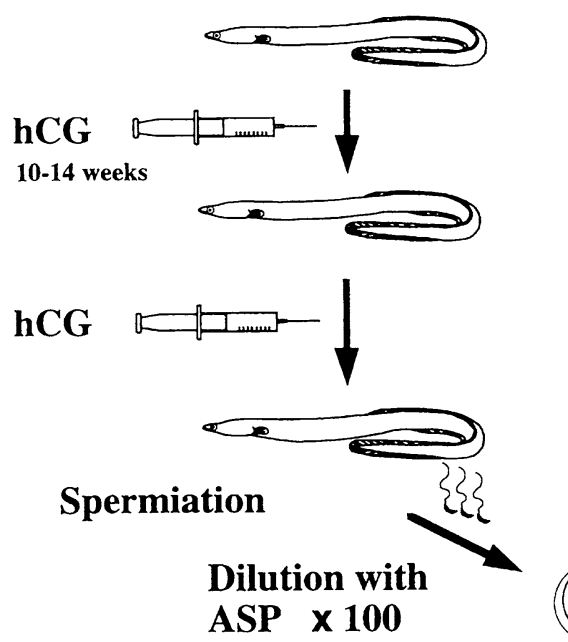

Female

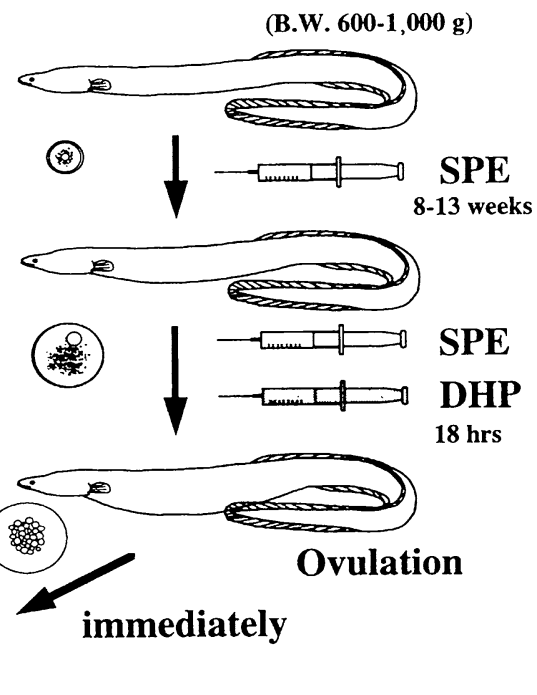

\section{Artificial fertilization}

Fig. 3 Summary of the artificial induction method of maturation and fertilization in the Japanese eel. SPE, salmon pituitary extract; DHP, 17, 20ß-dihydroxy-4-pregnen-3-one;

fertilization. Therefore, techniques for successful fertilization with a small quantity of inadequate quality of sperm need to be developed.

Recent progress of basic science on mechanism of spermatogenesis in the Japanese eel revealed that DHP is related to the regulation of sperm maturation (Miura et al. 2003). DHP increases $\mathrm{pH}$ in the seminal plasma, which in turn increases the sperm content of cAMP, thereby allowing the acquisition of sperm motility. Our data also indicate that injection of hCG affects the aqueous environment around the spermatozoa in the sperm duct and the potential for motility in the spermatozoa fluctuates with environmental factor(s) (Ohta and Unuma 2003). Milt with higher $\mathrm{pH}$ and potassium concentration of seminal plasma shows higher rate of motility in the spermiated male eel injected with hCG. From these fundamental researches, the effects of an artificial seminal plasma (ASP), which was produced based on the measurements of seminal plasma, on acquisition of the potential for motility of spermatozoa were examined. After immotile spermatozoa from testis were incubated with ASP in vitro, significant increase of the motility rate is observed (Ohta and Unuma 2003). Moreover, the rate of motility of the spermatozoa was
ASP, artificial seminal plasma; hCG, human chorionic gonadotropin. Slightly modified Ohta et al. (1997) with permission

controlled by adjusting the concentration of potassium ions and hydrogen bicarbonate ions in ASP. Therefore, from these studies, diluted spermatozoa with the ASP were stocked in refrigerator one day before fertilization and artificial fertilization was performed using the diluted spermatozoa and ovulated eggs (Fig. 3).

\section{Techniques for larval rearing}

Fertilized eggs begin to hatch about $40 \mathrm{~h}$ after fertilization at a water temperature of $22-23^{\circ} \mathrm{C}$. By 7 days after hatching, several marked changes are observed, such as pigmentation of eye and synthesis of digestive enzymes in the pancreas, suggesting that larvae acquire the ability to take feeds and digest them (Kurokawa et al. 1995). We first reported that preleptocephalus larvae at 13 day after hatching ingested rotifers, the most common initial feed used in the production of marine fish fry (Tanaka et al. 1995). However, even the larvae that ingest rotifers do not grow much larger and their survival period does not extend. Among feed items examined, finally, we found that eel larvae actively ingest a slurry-type diet 
made from shark egg powder. Eel larvae grow up from $3.7 \mathrm{~mm}$ long at just after hatch to about $8.1 \mathrm{~mm}$ in total length and the survival rate was $56 \%$ on 18th day. Therefore, we newly mixed soybean peptide, extract of krill, vitamin and mineral mixture to shark egg powder and made slurry diet. The eel larvae continued to survive and grow up linearly up to 50 days after hatching, their total length attained about $16 \mathrm{~mm}$ on the day and body depth gradually increased to yield the willow leaf-like form typical of wild leptocephali (Tanaka et al. 2001). Thereafter, larvae fed on further modified diet become fully grown leptocephali $50-60 \mathrm{~mm}$ in TL and have begun to metamorphose into glass eels approximately 250 after hatching (Tanaka 2003) (Fig. 4).

\section{Conclusion}

We developed the method for induction of maturation and ovulation in the Japanese eel by using the maturation-inducing steroid (DHP). In the male eels, in vitro incubation of the immotile spermatozoa with ASP shows significant increase of the motility rate. However, there are major differences in the extent of egg quality of the female eels induced in individual fish after the same hormonal treatments. The reason of inadequate quality of egg have not been clarified. Recent progress of researches on hypothalamus (gonadotropin-releasing hormone, GnRH)-pituitary (gonadotropin) axis (Suetake et al. 2003) and mechanisms of steroid production in eel ovary (Adachi et al. 2003) would facilitate to develop new techniques for inducing maturation of the Japanese eel. For example, synthetic GnRH will be able to use effectively for inducing sexual maturation of the Japanese eel, since they are effective in inducing sexual maturation in several commercially important fish (Matsuyama et al. 1995; Kumakura et al. 2003), including eels (Hirose 1992). Moreover, biotechnology for production of recombinant eel gonadotropins (Kamei et al. 2003) would be also useful tools for controlling all processes of spermatogenesis and oogenesis normally.

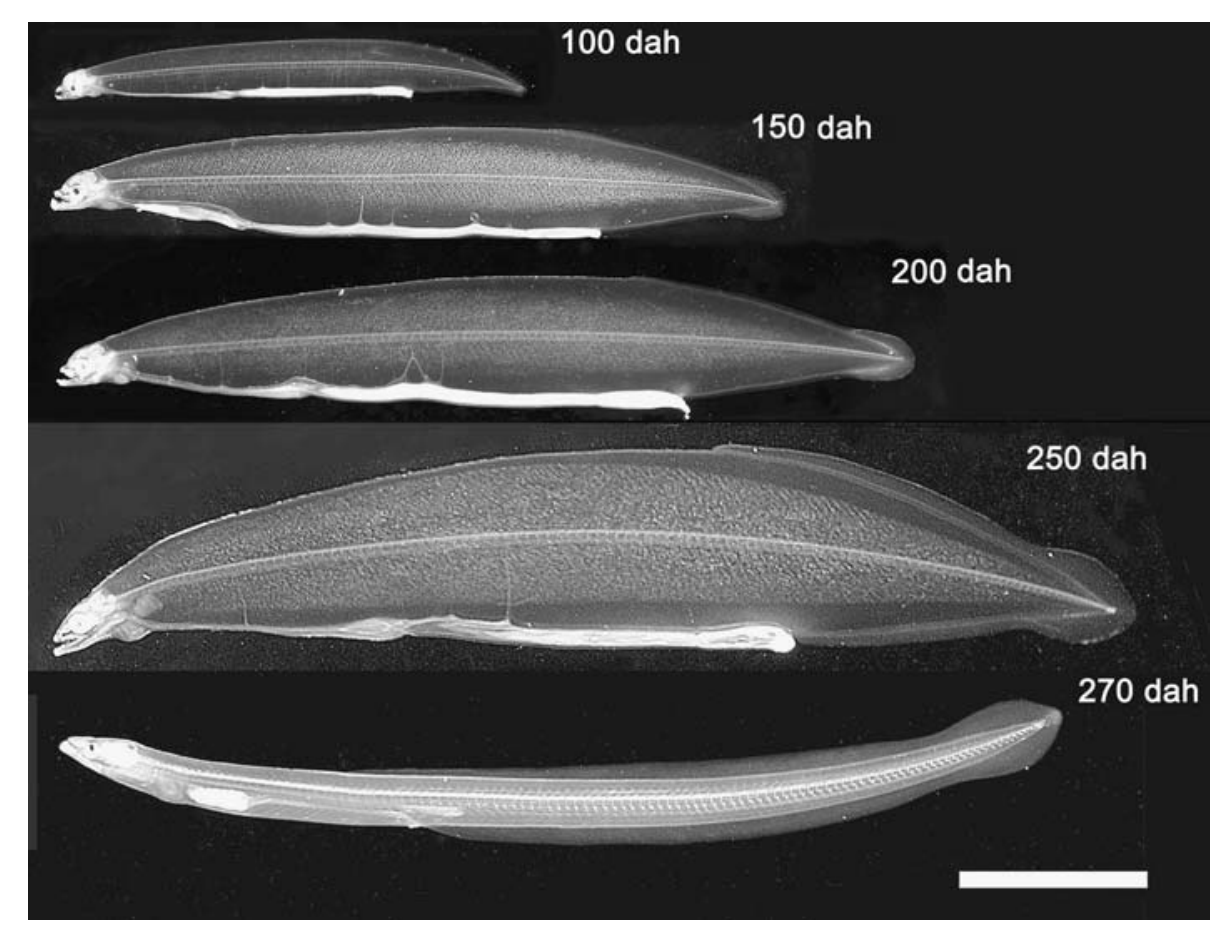

Fig. 4 Growth of captive-bred Anguilla japonica larvae. Age in days after hatching (dah), total length: $100 \mathrm{dah}, 24.8 \mathrm{~mm}$; $150 \mathrm{dah}, 39.0 \mathrm{~mm} ; 200 \mathrm{dah}, 45.1 \mathrm{~mm} ; 250 \mathrm{dah}, 57.1 \mathrm{~mm}$;
270 dah (glass eel), $52.6 \mathrm{~mm}$. Scale bar=10 mm. Reproduced from Tanaka (2003) with permission 


\section{References}

Adachi S, Ijiri S, Kazeto Y, Yamauchi K (2003) Oogenesis in the Japanese eel, Anguilla japonica. In: Aida K, Tsukamoto K, Yamauchi K (eds) Eel biology. Blackwell Publishing Asia, Melbourne, pp 301-317

Hirose K (1992) Induced spawning of Japanese eel with LHRH-A copolymer pellet. NOAA Tech Rep NMFS 106:43-48

Ijiri S, Kazeto Y, Takeda N, Chiba H, Adachi S, Yamauchi K (1995) Changes in serum steroid hormones and steroidogenic ovarian follicles during artificial maturation of cultivated Japanese eel, Anguilla japonica. Aquaculture $135: 3-16$

Kagawa H (2003) Artificial induction of oocyte maturation and ovulation. In: Aida K, Tsukamoto K, Yamauchi K (eds) Eel biology. Blackwell Publishing Asia, Melbourne, pp 401-414

Kagawa H, Iinuma N, Tanaka H, Ohta H, Okuzawa K (1998) Effects of rearing period in seawater on induced maturation in female Japanese eel Anguilla japonica. Fish Sci 64:77-82

Kagawa H, Kobayashi M, Hasegawa Y, Aida K (1994a) Insulin and insulin-like growth factors I and II induce final maturation of oocytes of red seabream, Pagrus major, in vitro. Gen Comp Endocrinol 95:293-300

Kagawa H, Tanaka H, Okuzawa K, Hirose K (1994b) Development of maturational competence of oocytes of red seabream, Pagrus major, after human chorionic gonadotropin treatment in vitro requires RNA and protein synthesis. Gen Comp Endocrinol 94:199-206

Kagawa H, Tanaka H, Ohta H, Okuzawa K, Hirose K (1995) In vitro effects of $17 \alpha$-hydroxyprogesterone and $17 \alpha$, $20 \beta$-dihydroxy-4-pregnen-3-one on final maturation of oocytes at various developmental stages in artificially matured Japanese eel Anguilla japonica. Fish Sci 61:1012-1015

Kagawa H, Tanaka H, Ohta H, Okuzawa K, Iinuma N (1997) Induced ovulation of 17, 20 $\beta$-dihydroxy-4-pregnen-3-one in the artificially matured Japanese eel, with special reference to ovulation time. Fish Sci 63:356-367

Kagawa H, Tanaka H, Unuma T, Ohta H, Gen K, Okuzawa K (2003) Role of prostaglandin in the control of ovulation in the Japanese eel Anguilla japonica. Fish Sci 69:234241

Kamei H, Ohira T, Yoshiura Y, Uchida N, Nagasawa H, Aida K (2003) Expression of a biologically active recombinant follicle stimulating hormone of Japanese eel Anguilla japonica using methylotropic yeast, Pichia pastoris. Gen Comp Endocrinol 134:2444-2254

Kumakura N, Okuzawa K, Gen K, Kagawa H (2003) Effects of gonadotropin-releasing hormone agonist and dopamine antagonist on hypothalamus-pituitary-gonadal axis of prepubertal female red seabream (Pagurs major). Gen Comp Endocrinol 131:264-273

Kurokawa H, Kagawa H, Ohta H, Tanaka H, Okuzawa K, Hirose K (1995) Development of digestive organs and feeding ability in larvae of Japanese eel (Anguilla japonica). Can J Fish Aquat Sci 52:1030-1036
Matsuyama M, Takeuchi H, Kashiwagi M, Hirose K, Kagawa $\mathrm{H}$ (1995) Induced gonadal development and spawning of immature red sea bream Pagurus major with LHRH-a administration in different ways during winter season. Fish Sci 61:472-477

Miura T, Miura C, Yamauchi K (2003) Spermatogenesis in the Japanese eel. In: Aida K, Tsukamoto K, Yamauchi K (eds) Eel biology. Blackwell Publishing Asia, Melbourne, pp 319-329

Miura T, Yamauchi K, Nagahama Y, Takahashi H (1991) Induction of spermatogenesis in male Japanese eel, Anguilla japonica, by a single injection of human chorionic gonadotropin. Zool Sci 8:63-73

Nagahama Y, Adachi S (1985) Identification of a maturationinducing steroid in a teleost, the amago salmon (Oncorhynchus rhodurus). Dev Biol 109:428-435

Nagahama Y, Yamashita M, Tokumoto T, Katsu Y (1995) Regulation of oocyte maturation in fish. Curr Top Dev Biol 30:103-145

Ohta H, Kagawa H, Tanaka H, Okuzawa K, Hirose K (1996a) Changes in fertilization and hatching rates with time after ovulation induced by 17, 20ß-dihydroxy-4-pregnen-3-one in the Japanese eel, Anguilla japonica. Aquaculture 139:291-301

Ohta H, Kagawa H, Tanaka H, Okuzawa K, Hirose K (1996b) Milt production in the Japanese eel Anguilla japonica induced by repeated injections of human chorionic gonadotropin. Fish Sci 62:44-49

Ohta H, Kagawa H, Tanaka H, Okuzawa K, Inuma N, Hirose K (1997) Artificial induction of maturation and fertilization in the Japanese eel, Anguilla japonica. Fish Physiol Biochem 17:163-169

Ohta H, Kagawa H, Tanaka H, Unuma T (2001) Control by the environmental concentration of ions of the potential for motility in Japanese eel spermatozoa. Aquaculture 198:339-351

Ohta H, Tanaka H (1997) Relationship between serum levels of human chorionic gonadotropin (hCG) and 11-ketotestosterone after a single injection of hCG and induced maturity in the male Japanese eel, Anguilla japonica. Aquaculture 153:123-134

Ohta H, Unuma T (2003) Induction of sperm maturation. In: Aida K, Tsukamoto K, Yamauchi K (eds) Eel biology. Blackwell Publishing Asia, Melbourne, pp 415-423

Patino R, Yoshizaki G, Thomas P, Kagawa H (2001) Gonadotropic control of ovarian follicle maturation: the twostage concept and its mechanisms. Com Biochem Physiol 129B:427-439

Suetake H, Okubo K, Yoshiura Y, Aida K (2003) GTH and GnRH molecules and their expression in the Japanese eel. In: Aida K, Tsukamoto K, Yamauchi K (eds) Eel biology. Blackwell Publishing Asia, Melbourne, pp $351-372$

Tanaka H (2003) Techniques for larval rearing. In: Aida K, Tsukamoto K, Yamauchi K (eds) Eel biology. Blackwell Publishing Asia, Melbourne, pp 427-434

Tanaka H, Kagawa H, Ohta H (2001) Production of leptocephali of Japanese eel (Anguilla japonica) in captivity. Aquaculture 201:55-60 
Tanaka H, Kagawa H, Ohta H, Okuzawa K, Hirose K (1995) The first report of eel larvae ingesting rotifers. Fish Sci 61:171-172

Yamauchi K, Nakamura M, Takahashi H, Takano K (1976) Cultivation of larvae of Japanese eel. Nature 263:412

Yamamoto K, Yamauchi K (1974) Sexual maturation of Japanese eel and production of eel larvae in the aquarium. Nature 251:220-222
Yamauchi K, Yamamoto K (1982) Experiments on artificial maturation and fertilization of the Japanese eel (Anguilla japonica). In: Richter CJ, Goos GJTh (eds) Reproductive physiology of fish. Pudoc Press, Wargeningen, pp 185189 